MATHEMATICS OF COMPUTATION

Volume 75 , Number 254 , Pages 767-790

S 0025-5718(06)01822-9

Article electronically published on January 23, 2006

\title{
OPTIMAL FILTER AND MOLLIFIER FOR PIECEWISE SMOOTH SPECTRAL DATA
}

\author{
JARED TANNER
}

This paper is dedicated to Eitan Tadmor for his direction

\begin{abstract}
We discuss the reconstruction of piecewise smooth data from its (pseudo-) spectral information. Spectral projections enjoy superior resolution provided the function is globally smooth, while the presence of jump discontinuities is responsible for spurious $\mathcal{O}(1)$ Gibbs' oscillations in the neighborhood of edges and an overall deterioration of the convergence rate to the unacceptable first order. Classical filters and mollifiers are constructed to have compact support in the Fourier (frequency) and physical (time) spaces respectively, and are dilated by the projection order or the width of the smooth region to maintain this compact support in the appropriate region. Here we construct a noncompactly supported filter and mollifier with optimal joint time-frequency localization for a given number of vanishing moments, resulting in a new fundamental dilation relationship that adaptively links the time and frequency domains. Not giving preference to either space allows for a more balanced error decomposition, which when minimized yields an optimal filter and mollifier that retain the robustness of classical filters, yet obtain true exponential accuracy.
\end{abstract}

\section{INTRODUCTION}

The Fourier projection of a $2 \pi$ periodic function

$$
S_{N} f(x):=\sum_{|k| \leq N} \hat{f}_{k} e^{i k x}, \quad \hat{f}_{k}:=\frac{1}{2 \pi} \int_{-\pi}^{\pi} f(x) e^{-i k x} d x,
$$

enjoys the well-known spectral convergence rate, that is, the convergence rate is as rapid as the global smoothness of $f(\cdot)$ permits. Specifically, if $f(\cdot)$ has $s$ bounded derivatives, then $\left|S_{N} f(x)-f(x)\right| \leq$ Const $\|f\|_{C^{s}} \cdot N^{1-s}$, and if $f(\cdot)$ is analytic, $\left|S_{N} f(x)-f(x)\right| \leq$ Const $\cdot e^{-\eta_{f} N}$. In the dual (frequency) space the global smoothness and spectral convergence are reflected in rapidly decaying Fourier coefficients $\left|\hat{f}_{k}\right| \leq 2 \pi k^{-s}\|f\|_{C^{s}}$. On the other hand, spectral projections of piecewise smooth functions suffer from the well-known Gibbs' phenomena, where the uniform convergence of $S_{N} f(x)$ is lost in the neighborhood of discontinuities. Moreover, the convergence rate away from the discontinuities deteriorates to first order.

Received by the editor May 19, 2004 and, in revised form, January 29, 2005.

2000 Mathematics Subject Classification. Primary 41A25, 42A10, 42A16, 42A20, 42C25, $65 \mathrm{~B} 10,65 \mathrm{~T} 40$.

Key words and phrases. Fourier series, filters, time-frequency localization, piecewise smooth, spectral projection.

The author was supported in part by NSF Grants DMS 01-35345 and 04-03041.

(C)2006 American Mathematical Society Reverts to public domain 28 years from publication 
Two interchangeable processes for recovering the rapid convergence associated with globally smooth functions are filtering, $\sigma(\cdot)$, in the dual space and mollification, $\psi(\cdot)$, in the physical space

$$
S_{N} f^{\sigma}(x):=\sum_{|k| \leq N} \sigma(k, N) \hat{f}_{k} e^{i k x} \equiv \psi * S_{N} f(x)=\int_{-\pi}^{\pi} \psi(y) S_{N} f(x-y) d y .
$$

For a filter defined in the Fourier (dual) space its associated mollifier is given by

$$
\psi(x):=\frac{1}{2 \pi} \sum_{k=-\infty}^{\infty} \sigma(k, N) e^{i k x},
$$

whereas for a mollifier defined in the physical space its associated filter's samples are the mollifier's Fourier coefficients, $\sigma(k, N):=\hat{\psi}_{k}$. Although filters and mollifiers have been have been studied extensively (see [14] and references therein), a number of fundamental questions remain:

- What are the constraints to achievable accuracy for a filtered or mollified Fourier projection?

- How close to reaching this achievable limit are the host of already developed filters and mollifiers?

- How has the historical construction of filters and mollifiers affected their properties?

- What is a filter and mollifier which achieves the optimal pointwise convergence rate?

In 92 a new error decomposition is introduced which is equally valid for filters and mollifiers. With this decomposition the fundamental competing errors are rendered transparent, and a unified analysis of filters and mollifiers is made available. The classical constructions of compactly supported filters and mollifiers are shown not to balance the fundamental competing errors, and as a result fail to approach the achievable accuracy. Specifically, it has been shown in 23 and 24] that even with optimally varying spatial orders of accuracy, compactly supported infinitely differentiable filters and mollifiers can at most obtain root exponential accuracy. That is, $\left|f(x)-\psi * S_{N} f(x)\right| \leq$ Const $_{f} \exp \left(-c(N d(x))^{1 / \alpha}\right)$ for $\alpha>1$, where $d(x)$ is the distance from the point being recovered to the nearest discontinuity. In $₫ 3$ and $\$ 4$ we abandon compact support, and by balancing the overall competing errors construct a filter and mollifier (Theorems 3.3 and 4.1, respectively) which obtain the optimal achievable rate of true exponential accuracy, $\left|f(x)-\psi * S_{N} f(x)\right| \leq$ Const $_{f} \exp (-c N d(x))$. This optimal accuracy is obtained via a joint time-frequency localization of the filter and mollifier, and results in a new adaptive scaling relationship $\sqrt{N / d(x)}$.

Filtering remains the most prevalent approach for the resolution of Gibbs' phenomenon due to its extraordinary robustness and the computational efficiency of FFTs. Applications are ubiquitous, including fields as varied as image processing and spectral viscosity methods in the solution of time dependent problems. However, filtering as stated in (1.2) corresponds to a symmetrid 1 localization about the

\footnotetext{
${ }^{1}$ Here we consider symmetric mollifiers and real-valued filters where considering a nonsymmetric region of smoothness yields error bounds tighter only by a constant factor. We note in passing the one-sided filters introduced in 4] where complex-valued filters are utilized in order to recover high order accuracy up to the discontinuities. Rather than such extrapolatory filters we focus on resolving the achievable accuracy of symmetric reconstructions.
} 
point being approximated. Unfortunately, this symmetric nature necessitates diminished accuracy when approaching a discontinuity. Other techniques have been developed to overcome Gibbs' phenomenon, recovering high order accuracy up to the discontinuities; e.g., [3, 5, 6, 12, 14, 19, and the references therein. The improved accuracy of these methods however comes at an increased computational cost of at least $\mathcal{O}\left(N^{2}\right)$. A practical procedure to overcome the limitations of filtering and yet retain the computational efficiency $\mathcal{O}(N \log N)$ is to use filters away from the discontinuities and a reprojection method such as Gegenbauer postprocessing near the discontinuities, 8 . The efficiency of this hybrid approach is in a large part determined by the size of the smooth region well approximated by the filtered Fourier projection. The exponential accuracy of the optimal filter and mollifier further decrease the computational cost of the hybrid method. In addition to the optimal filter and mollifier being useful tools in their own right, the analysis put forth in $₫ 2$ resolves the fundamental questions concerning filtering posed earlier.

\section{FUNDAMENTAL COMPETING ERRORS FOR FILTERS AND MOLLIFIERS}

The fundamental constraints to the achievable accuracy of a filtered Fourier sum are due to the limited information in the function's spectral projection and the mollifier-induced blurring of unrelated information across discontinuities. To take into account the discontinuity location in a straightforward manner we undertake the error analysis in the physical space. Toward separating the fundamental error contributions Gottlieb and Tadmor introduced the decomposition 2

$$
\begin{aligned}
E(N, x) & :=f(x)-\psi * S_{N} f(x) \\
& =(f(x)-\psi * f(x))+\left(\psi-S_{N} \psi\right) *\left(f(x)-S_{N} f(x)\right) \\
& =: R(N, x)+L_{D}(N, x),
\end{aligned}
$$

which we refer to as the regularization and dual space localization errors, respectively. The dual space localization error contains the effects of the limited information in the function's spectral projection, and is controlled by the dual space localization of the mollifier to the space of the given data, $[-N, N]$.

To separate the error caused by the blurring of information across discontinuities, we further decompose the regularization error. In particular, we segment the action of the mollifier in the largest symmetric region of smoothness, $[x-d(x), x+d(x)]$, where $d(x)$ is the distance from the point being recovered to the nearest discontinuity 3 i.e.,

$$
d(x):=\operatorname{dist}(x, \operatorname{sing} \operatorname{supp} f) .
$$

This decomposes 4 the regularization error into the accuracy and physical space localization errors, respectively,

$$
\begin{aligned}
R(N, x) & =\int_{|y| \leq d(x)}[f(x)-f(x-y)] \psi(y) d y+\int_{d(x) \leq|y| \leq \pi}[f(x)-f(x-y)] \psi(y) d y \\
& =: A(N, x)+L_{P}(N, x) .
\end{aligned}
$$

\footnotetext{
${ }^{2}$ This is achieved by adding and subtracting $\psi * f(x)$ from $\psi * S_{N} f(x)$ and $f(x)$, respectively, and noting the orthogonality of $S_{N} \psi$ and $f-S_{N} f$, [15.

${ }^{3}$ Discontinuities can be computed from a function's (pseudo-)spectral information in the efficient $\mathcal{O}(N \log N)$ computational cost, 10 11.

${ }^{4}$ The constant $f(x)$ is first multiplied by the integral of the unit mass mollifier, followed by segmenting the largest symmetric region of smoothness in the integral.
} 
The physical space localization error is determined by the decay of the mollifier for $d(x) \leq|y| \leq \pi$, and the accuracy error $A(N, x)$ is controlled by the number of local vanishing moments,

$$
\int_{-d(x)}^{d(x)} y^{j} \psi(y) d y \sim \delta_{j 0}, \quad j=0,1, \ldots, p-1,
$$

possessed by the mollifier as can readily be seen by Taylor expanding $f(x-y)$ about $x$, 14. Combining these components, the total error for a general filter or mollifier can be viewed in terms of the localization and the accuracy errors,

$$
E(N, x):=L_{D}(N, x)+L_{P}(N, x)+A(N, x) .
$$

The remainder of the analysis is given in terms of this decomposition which separates the competing fundamental error components: 1) the limited information in the given data, 2) the blurring of information across discontinuities, and 3) the accuracy of the new projection in terms of a local polynomial approximation. The competing nature of the localization errors is a result of the Fourier transform's property that a dilation of $\theta$ in one space corresponds to a contraction of $1 / \theta$ in the dual space. The accuracy error plays a more subtle role. As the number of vanishing moments is increased, the mollifier must possess an increasing number of oscillations, which in turn causes a broadening in the dual space. We now review the construction and properties of classical and adaptive filters in terms of the error decomposition (2.3).

Filters have traditionally been constructed with compact support, $\sigma(\xi)=0$ for $|\xi| \geq 1$, i.e., $\sigma(\xi) \in C_{0}[-1,1]$. Dilation by the projection order, $N$, then causes the filter to act only on the space of the given data, and as a result such filters have no dual space localization error. Moreover, a filter is said to be of order $p$ if it is sufficiently smooth, $\sigma \in C^{p}$, and satisfies the filter accuracy condition,

$$
\sigma^{(j)}(0) \sim \delta_{j 0}, \quad j=0,1, \ldots, p-1 .
$$

The filter's smoothness is reflected in the localization of its associated mollifier (1.3). Being well localized, the filter's accuracy condition implies that the mollifier possesses $p-1$ near vanishing local moments where the quality of the vanishing moments is determined by the mollifiers localization, 24. From these properties it has been shown that the accuracy and physical space localization errors are bounded by $\left\|\sigma^{(p)}\right\|_{L^{\infty}}\left\|f^{(p)}\right\|_{L^{\infty}}(N d(x))^{-p}$, 14, 25]. Examples of filters constructed to satisfy these conditions include: the 2nd order raised cosine, the 8th order sharpened raised cosine [14, the infinite order filter of Majda et al. [21], the spectral filter of Vandeven [25], and the adaptive filter of Tadmor and Tanner [24]. The adaptive order filter 24 balances the physical space localization and accuracy error components for general infinitely differentiable compactly supported filters. This was attained by analyzing the filter's smoothness in terms of the Gevrey regular spact5 and linking the filter order to the degree of the function's spectral projection, $N$, and $d(x)$.

\footnotetext{
${ }^{5} \mathrm{~A}$ function $\rho(\cdot)$ is in Gevrey regularity $\alpha$ if $\left\|\rho^{(s)}\right\|_{L^{\infty}} \leq K_{\rho}(s !)^{\alpha} \eta_{\rho}^{-s}$ for some $K_{\rho}, \eta_{\rho}$ independent of $s$. Analytic functions satisfy $\alpha=1$, and compactly supported functions can at most satisfy $\alpha>1$.
} 
Balancing the remaining two error contributions, $L_{p} \approx A$, results in the rootexponential convergence rate,

$$
\left|f(x)-S_{N} f^{\sigma}(x)\right| \leq \text { Const }_{f} \exp \left(-c(N d(x))^{1 / \alpha}\right)
$$

for $\alpha>1$.

Mollifiers, however, are traditionally defined to be compactly supported in the physical space, and, to avoid transferring information across discontinuities, are dilated by $d(x),[15$. As a result, there is no physical space localization error. To control the remaining dual space localization and accuracy errors, $p$ order mollifiers are constructed with $p-1$ vanishing moments, (2.3), and a similar order of smoothness, $\psi \in C^{p}$. The mollifier's physical space smoothness ensures dual space localization, and the vanishing moments control the accuracy error by being exact for polynomials of up to degree $p-1$. The spectral mollifier of Gottieb and Tadmor [15] was extended to the adaptive mollifier in [23, where the two remaining competing dual space and accuracy errors were balanced. Similar to the adaptive filter of [24, this was accomplished by considering Gevrey regularity and linking the mollifier order to $N$ and $d(x)$. The same root-exponential convergence rate, $\left|f(x)-\psi * S_{N} f(x)\right| \leq$ Const $_{f} \exp \left(-c(N d(x))^{1 / \alpha}\right)$ for $\alpha>1$, was likewise obtained.

Although compact support in either of the physical or dual spaces is desirable in some instances (as discussed in Remark 4.2), to achieve minimal error neither space has priority. By satisfying compact support, infinitely differentiable filters and mollifiers limit their accuracy to at most the root-exponential rates stated earlier. Additionally, even though the dilation factors $N$ and $d(x)$ for compactly supported filters and mollifiers, respectively, naturally appear to be performing the same action in the two different spaces, the Fourier transform implies that their dilation factors would be the inverse of one another. To overcome these limitations we abandon the traditional construction of compactly supported filters and mollifiers, and instead let the fundamental error decomposition (2.3) guide their construction. In particular, a filter and mollifier should possess the optimal joint time-frequency localization to $[x-d(x), x+d(x)] \times[-N, N]$ for a given number of near-vanishing moments. The minimal error is then achieved by equally balancing the three competing localization and accuracy errors, (2.3).

There exist a number of noncompactly supported filters, but their analysis has primarily been done by comparison with compactly supported filters. For example, the commonly used exponential filter, $\sigma(\xi)=\exp \left(-c \xi^{2 m}\right)$, satisfies the accuracy condition (2.4) for $p=2 m+1$, and the parameter $c$ is selected so that $\sigma( \pm 1)$ is at machine tolerance. Its accuracy is then justified by stating that when implemented numerically the computer treats it as being compactly supported. Other examples of noncompactly supported filters are the adaptive Erfc and Erfc-log filters introduced by Boyd, 2. These filters pioneered the concept of adaptive filters, yet lack a rigorous error analysis. Instead, their convergence is justified through numerical examples and by being asymptotic approximations of the Vandeven filter, [25, which satisfies the traditional properties of a compactly supported filter. The new error decomposition allows a framework for properly analyzing these and other noncompactly supported filters. The previously mentioned filters, however, are not known to possess optimal joint time-frequency localization in any sense. Moreover, their construction is not amenable to analysis in the physical space. Specifically, the Fourier transform of the exponential filter is given in terms of hypergeometric functions, and is not known for the Erfc and Erfc-log filters of Boyd. 
In lieu of carrying out the analysis for a general smooth (analytic) filter or an (exponentially) well localized mollifier, we focus on a specific filter-mollifier pair that possesses optimal joint time-frequency localization for a given number of near-vanishing moments. The Hermite Distributed Approximating Functionals, HDAFs, introduced in [18 satisfy these properties with the optimality of joint time-frequency localization measured by the classical uncertainty principle 6

$$
4 \pi\|x h(x)\|_{L^{2}} \cdot\|\xi(\mathcal{F} h)(\xi)\|_{L^{2}} \geq\|h\|_{L^{2}}^{2} .
$$

Definition 2.1 (HDAF, [18]). The $p$ order HDAF is given by

$$
\phi_{p, \gamma}(x):=e^{-x^{2} /\left(2 \gamma^{2}\right)} \gamma^{-1} \sum_{n=0}^{p} \frac{(-4)^{-n}}{n !} H_{2 n}\left(\frac{x}{\gamma \sqrt{2}}\right),
$$

where $H_{2 n}$ is the Hermite polynomia $\sqrt{7}$ of order $2 n$. The Fourier transform of (2.6) is given by

$$
\left(\mathcal{F} \phi_{p, \gamma}\right)(\xi):=e^{-\xi^{2} \gamma^{2} / 2} \sum_{n=0}^{p} \frac{\left(\xi^{2} \gamma^{2}\right)^{n}}{2^{n} n !} .
$$

Property 2.2 (HDAF localization, [18]). Let $\delta \phi_{p, \gamma}:=\left[\phi_{p, \gamma}-\phi_{p-1, \gamma}\right]$ be the difference between consecutive order HDAFs, and expand the time-frequency uncertainty in terms of the increase in frequency (dual) space uncertainty

$$
\begin{aligned}
\left\|x \phi_{p, \gamma}(x)\right\|_{L^{2}}^{2}( & \left\|\xi\left(\mathcal{F} \phi_{p-1, \gamma}\right)(\xi)\right\|_{L^{2}}^{2}+\left\|\xi\left(\mathcal{F} \delta \phi_{p, \gamma}\right)(\xi)\right\|_{L^{2}}^{2} \\
& \left.+2 R e \int_{-\infty}^{\infty} \xi^{2}\left(\mathcal{F} \phi_{p-1, \gamma}\right)(\xi)\left(\mathcal{F} \delta \phi_{p, \gamma}\right)(\xi) d \xi\right) .
\end{aligned}
$$

The $p$ order HDAF possesses joint minimal uncertainty in the sense that $\phi_{p, \gamma}(x)$ possesses the minimal increase in the variance, $\left\|\xi\left(\mathcal{F} \delta \phi_{p, \gamma}\right)(\xi)\right\|_{L^{2}}^{2}$, while also increasing the number of vanishing moments and holding constant

$$
\left\|x \phi_{p, \gamma}(x)\right\|_{L^{2}}^{2}=\left\|x \phi_{p-1, \gamma}(x)\right\|_{L^{2}}^{2} .
$$

Characteristic plots of HDAFs are presented in Figures 5.1 and 5.2

We now undertake a detailed error analysis of a filter, \$3, and mollifier, \$4, constructed from the HDAFs, by selecting the optimal values for the localization, $\gamma$, and accuracy, $p$, parameters. By balancing the joint time-frequency localization for the region

$$
[x-d(x), x+d(x)] \times[-N, N]
$$

a new fundamental scaling factor is determined, $\sqrt{N / d(x)}$. As in [23] and [24] the adaptive optimal number of near-vanishing moments, $p(N, d(x))$, is determined for this scaling relation, balancing the overall error. Having balanced the fundamental

\footnotetext{
${ }^{6}$ For consistency with the Fourier series expansion used here, (1.1), we use the Fourier transform normalization $(\mathcal{F} h)(\xi):=\frac{1}{2 \pi} \int_{-\infty}^{\infty} e^{i x \xi} h(x) d x$; note that equality in 2.5) is satisfied if and only if $h(x)=\exp \left(-c x^{2}\right)$ for $c>0$.

${ }^{7}$ Hermite polynomials are orthogonal under the Gaussian weight

$$
\int_{-\infty}^{\infty} e^{-x^{2}} H_{m}(x) H_{n}(x) d x=\sqrt{\pi} 2^{n} n ! \delta_{n m} .
$$
}


error contributions, the resulting optimal filter and mollifier introduced in $\$ 3$ and $\$ 4$ achieve substantially improved convergence rates over classical filters and mollifiers as stated in Theorems 3.3 and 4.1 and exhibited in the numerical examples, $\$ 5$.

\section{Optimal ADAPTIVE ORDER FILTER}

In this section we construct the optimal adaptive order filter from the family of HDAFs, Definition 2.1. by determining the localization, $\gamma$, and accuracy, $p$, parameters for

$$
\sigma_{\gamma}(\xi):=e^{-\xi^{2} \gamma^{2} / 2} \sum_{n=0}^{p} \frac{\left(\xi^{2} \gamma^{2}\right)^{n}}{2^{n} n !}
$$

We reiterate that this filter satisfies optimal joint time-frequency localization for the given order, $\sigma_{\gamma}^{(n)}(0)=\delta_{n, 0}$ for $n=0,1, \ldots, 2 p$. To bound the error after filtering a function's spectral projection with (3.1), we must determine bounds on the physical and dual space localization errors, as well as the accuracy error, (2.3). After constructing these bounds we will determine the optimal localization and moment parameters, $\gamma$ and $p$ respectively, by balancing the competing error component's decay rates. We begin with the dual space localization error which is bounded by

$$
\begin{aligned}
\left|L_{D}(N, x)\right| & :=\left\|\left(\psi-S_{N} \psi\right) *\left(f(x)-S_{N} f(x)\right)\right\|_{L^{\infty}} \\
& \leq\left\|f-S_{N} f\right\|_{L^{1}} \cdot\left\|\psi-S_{N} \psi\right\|_{L^{\infty}} \\
& \leq \text { Const }_{f} \cdot \sum_{k=N+1}^{\infty} \sigma_{\gamma}\left(\frac{k}{N}\right) \\
& \leq \text { Const }_{f} \cdot \int_{1}^{\infty} \sigma_{\gamma}(\xi) d \xi \\
& \leq \text { Const }_{f} \cdot \gamma^{-2} p^{2}\left(\frac{\gamma^{2} e}{2 p}\right)^{p} e^{-\gamma^{2} / 2},
\end{aligned}
$$

where Const $_{f}$ is a possibly different constant depending on $f$. Note that the quantity $\left(\gamma^{2} e / 2 p\right)^{p}$ is maximized at $p=\gamma^{2} / 2$, canceling the exponential decay, $\exp \left(-\gamma^{2} / 2\right)$, and as such, for exponential dual space localization to $[-N, N]$ the filter order must satisfy $p<\gamma^{2} / 2$.

We now turn to the physical space localization error which is controlled by the decay of the filter's associated mollifier. As the filter is dilated by the factor $N$ in the dual space, its associated mollifier is dilated by $1 / N$ in the physical space. Although we do not have an explicit representation of the mollifier formed from the filter's samples, (1.3), the mollifier is directly related to the filter's inverse Fourier transform. More precisely, the mollifier is constructed from the uniform sampling of the filter, and accordingly the Poisson summation formula states that the dual space sampling corresponds to physical space periodization of the filter's Fourier transform, (2.6),

$$
\psi(x) \equiv \sum_{m=-\infty}^{\infty} \phi_{p, \gamma}(N(x+2 \pi m))
$$


Before investigating the localization bound for the mollifier, we determine a bound on the filter's Fourier transform, (2.6). That is,

$$
\begin{aligned}
\left|\phi_{p, \gamma}(x)\right| & \leq e^{-x^{2} /\left(2 \gamma^{2}\right)} \gamma^{-1}\left|\sum_{n=0}^{p} \frac{(-4)^{-n}}{n !} H_{2 n}\left(\frac{x}{\gamma \sqrt{2}}\right)\right| \\
& \leq \text { Const } \cdot e^{-x^{2} /\left(4 \gamma^{2}\right)} \gamma^{-1} \kappa \sum_{n=0}^{p} \frac{\sqrt{2 n !}}{2^{n} n !} \\
& \leq \text { Const } \cdot \gamma^{-1} p^{3 / 4} e^{-x^{2} / 4 \gamma^{2}}
\end{aligned}
$$

where we utilized: 1) the bound on Hermite polynomials, $\left|H_{n}(x)\right|<1.1 \sqrt{n !} 2^{n / 2} e^{x^{2} / 2}$, and 2) Sterling's inequality, $1<n !(e / n)^{n}(2 \pi n)^{-1 / 2}<1+1 / 4 n$, [16]. From relationship (3.3) and the decay of $\phi_{p, \gamma}(x)$ we note that for $|x| \leq \pi$, the mollifier and the filter's Fourier transform are exponentially close,

$\left|\psi(x)-\phi_{p, \gamma}(N x)\right| \equiv\left|\sum_{m \neq 0} \phi_{p, \gamma}(N(x+2 \pi m))\right| \leq$ Const $\cdot \frac{\gamma p^{3 / 4}}{N^{2}} e^{-(\pi N / 2 \gamma)^{2}}, \quad|x| \leq \pi$,

and as a result they decay at the same exponential rate,

$$
|\psi(x)| \leq \text { Const } \cdot\left(1+\left(\frac{\gamma}{N}\right)^{2}\right)\left|\phi_{p, \gamma}(N x)\right| .
$$

Returning to the physical space localization error we obtain the bound,

$$
\begin{aligned}
\left|L_{P}(N, x)\right| & :=\left|\int_{d(x) \leq|y| \leq \pi}[f(x)-f(x-y)] \psi(y) d y\right| \leq 2\|f\|_{L^{\infty}} \int_{d(x)}^{\pi}|\psi(y)| d y \\
& \leq \text { Const }_{f}\left(1+(\gamma / N)^{2}\right) \int_{d(x)}^{\pi}\left|\phi_{p, \gamma}(N y)\right| d y \\
& \leq \text { Const }_{f} \cdot\left(1+(\gamma / N)^{2}\right) \frac{\gamma p^{3 / 4}}{N^{2} d(x)} e^{-(N d(x) / 2 \gamma)^{2}} .
\end{aligned}
$$

With the localization errors quantified we now turn to bounding the accuracy error by determining the number and quality of the mollifier's near vanishing moments. Due to the length of this calculation we segment it in the following subsection.

3.1. Accuracy error. Traditionally the accuracy error,

$$
A(N, x):=\int_{|y| \leq d(x)}[f(x)-f(x-y)] \psi(y) d y,
$$

is bounded by Taylor expanding $g_{x}(y):=[f(x)-f(x-y)]$ about $y=0$ and taking the largest symmetric region in which $g_{x}(y)$ is analytic, $|y| \leq d(x)$ where as stated earlier $d(x)$ is the distance from $x$ to the nearest discontinuity of $f(\cdot)$. The canonical Taylor expansion bound is then controlled by the vanishing moments and the truncation of the Taylor expansion, $d(x)^{q}\left\|g_{x}\right\|_{C^{q}} / q$ !. Here we focus on piecewise analyti 8 functions in which case the Cauchy integral formula quantifies

\footnotetext{
${ }^{8}$ Note that the analysis presented for the localization errors is the same for piecewise $C^{s}$ functions; however, the accuracy error is decreased, resulting in an overall accuracy $\mathcal{O}\left(N^{-s}\right)$ for piecewise $C^{s}$ functions.
} 
the regularity as

$$
\|f\|_{C^{s}[x-d(x), x+d(x)]} \leq \text { Const } \cdot \frac{s !}{\eta_{f}^{s}}
$$

where $f(x-z)$ extends analytically into the complex plane perpendicularly by the distance $\eta_{f}$. Incorporating the regularity bound in the classical $q-1$ term truncated Taylor expansion yields a truncation error proportional to $\left(d(x) / \eta_{f}\right)^{q}$ which is only decreasing for $d(x)<\eta_{f}$. For this reason, utilizing the maximum region of smoothness rather than selecting a small subinterval makes the Taylor expansion bound ineffective. Alternatively, we consider the near orthogonality of the mollifier and low order Chebyshev polynomials. Before investigating this approach in detail we first quantify the number and quality of the near vanishing moments for the mollifier associated with the filter (3.1).

Similar to the case of the physical space localization, we approach the local moments through the properties of the filter's Fourier transform, $\phi_{p, \gamma}(\cdot)$, which possesses $2 p+1$ exactly vanishing moments when taken over the entire real line. Here, however, we are interested in local symmetric moments, defined as

$$
M_{n, d(x)}:=\int_{-d(x)}^{d(x)} y^{n} \psi(y) d y, \quad d(x) \leq \pi .
$$

As the mollifier is even it possesses exactly vanishing odd moments. To control the even moments for $n \leq p$ we subtract the exact moment of $\phi_{p, \gamma}$ and segment the local region of smoothness, $|y| \leq d(x)$,

$$
\begin{aligned}
M_{2 n, d(x)} & =\int_{-d(x)}^{d(x)} y^{2 n} \psi(y) d y-\int_{-\infty}^{\infty} y^{2 n} \phi_{p, \gamma}(N y) d y \\
& =\int_{-d(x)}^{d(x)} y^{2 n}\left[\psi(y)-\phi_{p, \gamma}(N y)\right] d y-\int_{|y|>d(x)} y^{2 n} \phi_{p, \gamma}(N y) d y=: \mathcal{M}_{1}+\mathcal{M}_{2} .
\end{aligned}
$$

The first component is small due to the similarity of the mollifier and the filter's Fourier transform (3.5)

$$
\left|\mathcal{M}_{1}\right| \leq \max _{y \in[-\pi, \pi]}\left|\psi(y)-\phi_{p, \gamma}(N y)\right| \cdot 2 \int_{0}^{d(x)} y^{2 n} d y \leq \text { Const } \cdot \frac{\gamma p^{3 / 4}}{N^{2}} d(x)^{2 n} e^{-(\pi N / 2 \gamma)^{2}},
$$

and the second is controlled by the physical space localization (3.4)

$$
\begin{aligned}
\left|\mathcal{M}_{2}\right| & \leq \int_{|y|>d(x)} y^{2 n}\left|\phi_{p, \gamma}(N y)\right| d y \leq \text { Const } \cdot \gamma^{-1} p^{3 / 4} \int_{d(x)}^{\infty} y^{2 n} e^{-(y N / 2 \gamma)^{2}} d y \\
& \leq \text { Const } \cdot \frac{\gamma p^{3 / 4}}{N^{2} d(x)^{1 / 2}} e^{-(N d(x) / 2 \gamma)^{2}} n !\left(\frac{2 \gamma}{N}\right)^{2 n} \sum_{k=0}^{n} \frac{1}{k !}\left(\frac{N d(x)}{2 \gamma}\right)^{2 k} \\
& \leq \text { Const } \cdot \frac{\gamma p^{3 / 4} n}{N^{2} d(x)^{1 / 2}} d(x)^{2 n} e^{-(N d(x) / 2 \gamma)^{2}}, \quad n \leq\left(\frac{N d(x)}{2 \gamma}\right)^{2} .
\end{aligned}
$$

In the above bound for $\mathcal{M}_{2}$, the sum is initially increasing, reaching its maximum at $k=(N d(x) / 2 \gamma)^{2}$. As a result, for $n \leq(N d(x) / 2 \gamma)^{2}$ the sum reaches its maximum at $k=n$, canceling the term $n !(2 \gamma / N)^{2 n}$. Combining the bounds for $\mathcal{M}_{1}$ and $\mathcal{M}_{2}$, the number and quality of the moments is given by

$$
\left|M_{2 n, d(x)}\right| \leq \text { Const } \cdot \frac{p^{3 / 4} \gamma n}{N^{2} d(x)^{1 / 2}} d(x)^{2 n} e^{-(N d(x) / 2 \gamma)^{2}}, \quad n \leq\left(\frac{N d(x)}{2 \gamma}\right)^{2} .
$$


With near vanishing moments quantified in (3.7), we return to bounding the accuracy error, $A(N, x):=\int_{|y| \leq r}[f(x)-f(x-y)] \psi(y) d y$. Rather than the traditional Taylor expansion approach discussed above, we use a Chebyshev expansion of $g_{x}(y)$ over $y \in(-d(x), d(x))$ which gives a near min-max approximation for a given order polynomial. Before investigating this alternative Chebyshev polynomial-based error decomposition, we state the definition and properties for the Chebyshev polynomials which are relevant here.

Definition 3.1 (Chebyshev polynomials, 20]). The $k$ th order Chebyshev polynomial is given by

$$
T_{k}(x):=\sum_{l=0}^{\lfloor k / 2\rfloor} c_{l}^{(k)} x^{k-2 l}, \quad c_{l}^{(k)}:=(-1)^{l} 2^{k-2 l-1} \frac{k}{k-l} \cdot\left(\begin{array}{c}
k-l \\
l
\end{array}\right)
$$

and the Chebyshev expansion of a function, $h(\cdot)$, by

$$
S_{M}^{T} h(x):=\sum_{k=0}^{M} h_{k}^{T} T_{k}(x), \quad h_{k}^{T}:=\frac{2}{\pi} \int_{-1}^{1} \frac{T_{k}(x) h(x)}{\sqrt{1-x^{2}}}
$$

Property 3.2 (Chebyshev polynomials, bounds). The coefficients of a Chebyshev expansion of an analytic function decay exponentially, and as a result, Chebyshev projections converge at an exponential rate

$$
\left|h_{k}^{T}\right| \leq \text { Const }_{h} \cdot \beta_{h}^{-k} \quad \Longrightarrow \quad \max _{|x| \leq 1}\left|h(x)-S_{M}^{T} h(x)\right| \leq \operatorname{Const}_{h} \beta_{h}^{-M},
$$

where $\beta_{h}>1$ is a constant depending on the analytic extension of $h(\cdot)$ to the complex plane. Additionally, the classical three-term recursion relationship gives a bound on the growth of the coefficients composing a Chebyshev polynomial

$$
c_{l}^{(k+1)}=2 c_{l}^{(k)}-c_{l+1}^{(k-1)} \quad \Longrightarrow \quad\left|c_{l}^{(k)}\right| \leq(1+\sqrt{2})^{k} \quad \forall l .
$$

We now turn to bounding the accuracy error, $A(N, x)$, decomposed into the two terms

$$
\begin{aligned}
A(N, x)= & \int_{|y| \leq d(x)} \psi(y) g_{x}(y) d y \\
= & \int_{|y| \leq d(x)} \psi(y)\left(g_{x}(y)-S_{p}^{T}\left(g_{x}\right)(y)\right) d y \\
& +\int_{|y| \leq d(x)} \psi(y) S_{p}^{T}\left(g_{x}\right)(y) d y=: \mathcal{A}_{1}+\mathcal{A}_{2},
\end{aligned}
$$

where the first component is controlled by the exponential convergence of the Chebyshev projection, (3.8), of $g_{x}(y):=f(x)-f(x-y)$ which is analytic for $y \in(x-d(x), x+d(x))$,

$$
\left|\mathcal{A}_{1}\right| \leq \text { Const }_{f} \cdot \max _{|y| \leq d(x)}\left|g_{x}(y)-S_{p}^{T}\left(g_{x}\right)(y)\right| \int_{|y| \leq d(x)}|\psi(y)| d y \leq \text { Const }_{f} \cdot N d(x) \beta_{f}^{-p}
$$


The second component, $\mathcal{A}_{2}$, is small for modest $p$ due to the near-vanishing moments of the mollifier which correspond to near orthogonality with low order Chebyshev polynomials 9

$$
\begin{aligned}
& \left|\int_{|y| \leq d(x)} T_{2 k}\left(\frac{y}{d(x)}\right) \psi(y) d y\right| \\
& \quad \leq \sum_{l=0}^{k} d(x)^{2(l-k)}\left|c_{l}^{(2 k)}\right| \cdot\left|M_{2(k-l), d(x)}\right| \\
& \quad \leq(1+\sqrt{2})^{2 k} \sum_{j=0}^{k} d(x)^{-2 j}\left|M_{2 j, d(x)}\right| \\
& \quad \leq \text { Const } \cdot \frac{p^{3 / 4} k^{2} \gamma}{N^{2} d(x)^{1 / 2}}(1+\sqrt{2})^{2 k} e^{-(N d(x) / 2 \gamma)^{2}}, \quad k \leq\left(\frac{N d(x)}{2 \gamma}\right)^{2},
\end{aligned}
$$

resulting in the bound

$$
\begin{aligned}
\left|\mathcal{A}_{2}\right| & =\left|\int_{|y| \leq d(x)} \psi(y) S_{p}^{T}\left(g_{x}\right)(y) d y\right| \\
& =\left|\sum_{k=0}^{p}\left(g_{x}\right)_{k}^{T} \int_{|y| \leq d(x)} T_{k}\left(\frac{y}{d(x)}\right) \psi(y) d y\right| \\
& \leq \text { Const }_{f} \sum_{k=0}^{\lfloor p / 2\rfloor} \beta_{f}^{-2 k}\left|\int_{|y| \leq d(x)} T_{2 k}\left(\frac{y}{d(x)}\right) \psi(y) d y\right| \\
& \leq \text { Const }_{f} \frac{p^{15 / 4} \gamma}{N^{2} d(x)^{1 / 2}} e^{-(N d(x) / 2 \gamma)^{2}}\left(1+\left(\frac{1+\sqrt{2}}{\beta_{f}}\right)^{p}\right) .
\end{aligned}
$$

Combining the bounds for $\mathcal{A}_{1}$ and $\mathcal{A}_{2}$ yields the total accuracy error bound,

$$
\begin{aligned}
|A(N, x)| \leq \text { Const }_{f} \cdot N d(x) \beta_{f}^{-p} \\
\\
+ \text { Const }_{f} \frac{p^{15 / 4} \gamma}{N^{2} d(x)^{1 / 2}} e^{-(N d(x) / 2 \gamma)^{2}}\left(1+\left(\frac{1+\sqrt{2}}{\beta_{f}}\right)^{p}\right) .
\end{aligned}
$$

3.2. Composite error. Combining the bounds for the localization and accuracy error components $\left(L_{D}, L_{P}\right.$, and $A$ in (3.2), (3.6), and (3.9) respectively), we arrive at the overall error bound

$$
\left|f(x)-S_{N} f^{\sigma}(x)\right| \leq \text { Const }_{f}\left[N d(x) \beta_{f}^{-p}+C_{1} e^{-\gamma^{2} / 2}+C_{2} e^{-(N d(x) / 2 \gamma)^{2}}\right]
$$

with functions

$$
\begin{gathered}
C_{1}:=\left(\frac{p}{\gamma}\right)^{2}\left(\frac{\gamma^{2} e}{2 p}\right)^{p} \\
C_{2}:=\frac{\gamma p^{3 / 4}}{N^{2} d(x)}\left(1+\left(\frac{\gamma}{N}\right)^{2}\right)+\frac{p^{15 / 4} \gamma}{N^{2} d(x)^{1 / 2}}\left(1+\left(\frac{1+\sqrt{2}}{\beta_{f}}\right)^{p}\right) .
\end{gathered}
$$

\footnotetext{
${ }^{9}$ We only consider the even Chebyshev polynomials as the mollifier is even, giving exact orthogonality to all odd functions.
} 
The optimal behavior of the localization and accuracy parameters, $\gamma$ and $p$ respectively, are selected so that the competing error components possess the same decay rate, with the dominant error components given by $\beta_{f}^{-p}$, $\exp \left(-\gamma^{2} / 2\right)$, and $\exp \left(-(N d(x) / 2 \gamma)^{2}\right)$. Although the optimal values of $\gamma$ and $p$ depend on the particular function being approximated through $\beta_{f}$, the decay rates can be balanced by equating the exponents, $p=\gamma^{2} / 2=(N d(x) / 2 \gamma)^{2}$, resulting in

$$
\gamma:=\sqrt{\alpha N d(x)}, \quad p:=\kappa N d(x) .
$$

With these relationships we return to the second and third components of the overall error, as expressed in equation (3.10), fully including the growth rate of $C_{1}$ and $C_{2}$. For the parameters selected as described in (3.12), the second component is bounded by

$$
C_{1} e^{-\gamma^{2} / 2}=\frac{\kappa^{2}}{\alpha} N d(x)\left(\frac{2 \kappa^{\kappa} e^{\alpha / 2}}{(\alpha e)^{\kappa}}\right)^{-N d(x)}
$$

and the third error element simplifies to

$$
\begin{aligned}
C_{2} e^{-(N d(x) / 2 \gamma)^{2}} & \leq \text { Const } \cdot d(x)^{15 / 4} N^{9 / 4}\left(1+\left(\frac{\beta_{f}}{1+\sqrt{2}}\right)^{-\kappa N d(x)}\right) e^{-\frac{1}{2 \alpha} N d(x)} \\
& \leq \text { Const } \cdot d(x)^{15 / 4} N^{9 / 4}\left(\frac{e^{1 / 2 \alpha}}{(1+\sqrt{2})^{\kappa}}\right)^{-N d(x)}
\end{aligned}
$$

where the second line is due to $\beta_{f}>1$ for $f(\cdot)$ piecewise analytic. Exponential decay in $N d(x)$ of the second and third component requires $(\alpha e / \kappa)^{\kappa} / 2 e^{\alpha / 2}<1$ and $\exp (1 / 2 \alpha)(1+\sqrt{2})^{-\kappa}>1$, respectively.

The above bounds are summarized in the following theorem:

Theorem 3.3. Given the $N$ truncated Fourier coefficients, of a piecewise analytic function, $\left\{\hat{f}_{k}\right\}_{|k| \leq N}$, the function can be recovered within the exponential bound

$$
\left|f(x)-\frac{1}{2 \pi} \sum_{|k| \leq N} \sigma_{\mathrm{opt}}(k, N, x) \hat{f}_{k} e^{i k x}\right| \leq \operatorname{Const}_{f} \cdot N^{9 / 4} \tau^{-N d(x)}
$$

where $\tau:=\min \left(\beta_{f}^{k}, 2 e^{\alpha / 2}\left(\frac{\kappa}{\alpha e}\right)^{\kappa}, \frac{e^{1 / 2 \alpha}}{(1+\sqrt{2})^{\kappa}}\right)$ and with the adaptive filter

$$
\sigma_{\text {opt }}(k, N, x):=e^{\frac{-\alpha k^{2} d(x)}{2 N}} \sum_{n=0}^{\lfloor\kappa N d(x)\rfloor} \frac{1}{n !}\left(\frac{\alpha k^{2} d(x)}{2 N}\right)^{n} .
$$

Although the optimal values for the free constants, $\alpha$ and $\kappa$, depend on the function's regularity constant, $\beta_{f}$, convergence is ensured, i.e., $\tau>1$ when $\alpha \kappa<$ $1 / 2 \ln (1+\sqrt{2}) \approx 0.56$. We conclude this section on the optimal filter for truncated Fourier series with remarks concerning practical numerical computations and the optimal filter's dilation relationship. 
Remark 3.4. Notwithstanding that the optima 10 values for the free constants, $\alpha$ and $\beta$, are function-dependent, practical function-independent values that can be realized through a mix of numerical experiments and a rough balancing of the primary terms. In particular, numerical experiments encourage a relatively small value for $\kappa$, making the dominant contributions from the localization constant given by $e^{\alpha / 2}$ and $e^{1 / 2 \alpha}$, which are balanced for $\alpha=1$. Numerical experiments furthermore indicate that selecting $\kappa=1 / 15$ gives good results for a variety of functions with significantly different regularity constants. In this case, $\tau$ reduces to

$$
\tau=\min \left(1.55, \beta_{f}^{1 / 15}\right) \quad \text { with } \quad \alpha=1, \quad \kappa=1 / 15 .
$$

We reiterate that the above parameter selection is not optimal, but note that it does guarantee exponential convergence for $f(x)$ piecewise analytic. A larger value for the accuracy constant, $\kappa$, is encouraged for functions which are more oscillatory, resulting in smaller values of $\beta_{f}$.

Remark 3.5. In contrast to classical filters which are dilated by $N$ to maintain compact support in $[-N, N]$, the optimal filter is dilated by $\sqrt{N / d(x)}$ to achieve optimal joint time-frequency localization to the domain $[x-d(x), x+d(x)] \times[-N, N]$. This adaptive time-frequency localization is a consequence of the localization parameter $\gamma \sim \sqrt{N d(x)}$. In $\$ 4$ we construct the optimal mollifier defined in the physical space, arriving at the dilation rate $\sqrt{d(x) / N}$. Again, this is in contrast to traditional compactly supported mollifiers which are dilated by $d(x)$ so as to avoid transferring information across the discontinuity. For filtering and mollification to be performing the same action in dual spaces, the Fourier transform implies that their dilation parameters should be the inverse of one another. This is indeed the case for the optimal filter and mollifier, and is not satisfied by classical compactly supported constructions, which as stated before are traditionally dilated by $N$ and $d(x)$, respectively. In addition to recovering true exponential accuracy, this dilation property is one of the core advances of the optimal filter and mollifier.

3.3. Filtering of pseudo-spectral data. The discrete analog to a function's spectral projection, $S_{N} f$, is its pseudo-spectral information, that is its equidistant samples, $f\left(y_{\nu}\right)$, where $y_{\nu}:=\frac{\pi}{N}(\nu-N)$ for $\nu=0,1, \ldots, 2 N-1$. The function's trigonometric interpolant is formed from these samples,

$$
I_{N} f(x):=\sum_{|k| \leq N} \tilde{f}_{k} e^{i k x}, \quad \tilde{f}_{k}:=\frac{\pi}{N} \sum_{\nu=0}^{2 N-1} f\left(y_{\nu}\right) e^{-i k y_{\nu}},
$$

where the pseudo-spectral coefficients, $\tilde{f}_{k}$, are an approximation of the true Fourier coefficient, $\hat{f}_{k}$; replacing the integral in (1.1) with its trapezoidal sum. Although for piecewise smooth functions the trapezoidal quadrature is only first order, $\mid \tilde{f}_{k}-$ $\hat{f}_{k} \mid \approx \mathcal{O}\left(N^{-1}\right)$, the function $f(\cdot)$ can be approximated from its pseudo-spectral data within the same bound as presented in Theorem 3.3 .

\footnotetext{
${ }^{10}$ Here we concern ourselves with near-function-independent estimates of the parameters $\alpha$ and $\kappa$, but note that the convergence rate for a given function may be increased if $\beta_{f}$ were approximated, [9].
} 
An error decomposition similar to (2.3) is satisfied for the filtered trigonometric interpolant,

$$
\begin{aligned}
E^{p s}(N, x) & :=f(x)-\psi * I_{N} f(x) \\
& =(f(x)-\psi * f(x))+\left(\psi-S_{N} \psi\right) *\left(f(x)-I_{N} f(x)\right) \\
& =: R(N, x)+L_{D}^{p s}(N, x) \equiv L_{D}^{p s}(N, x)+L_{P}(N, x)+A(N, x),
\end{aligned}
$$

where the physical space localization and accuracy errors are unchanged from the spectral projection error expansion. The essential component of the dual space localization error is the decay of $\left\|\Psi-S_{N} \Psi\right\|_{L^{\infty}}$, which is unchanged. As such, the modified error $L_{D}^{p s}$ satisfies the same bound as in the case of the spectral projection, (3.2), with $\left\|f-S_{N} f\right\|_{L^{1}}$ replaced by $\left\|f-I_{N} f\right\|_{L^{1}}$, which are of the same order. With the same bounds satisfied for the filtered trigonometric interpolant, the optimal filter is selected as before in Theorem 3.3 resulting in the following theorem:

Theorem 3.6. Given the $2 N$ equidistant samples of a piecewise analytic function, $\left\{f\left(\frac{\pi}{N}(\nu-N)\right)\right\}_{\nu=0}^{2 N-1}$, by filtering the trigonometric interpolant, (3.16), the function can be recovered within the exponential bound

$$
\left|f(x)-\frac{1}{2 \pi} \sum_{|k| \leq N} \sigma_{\text {opt }}(k, N, x) \tilde{f}_{k} e^{i k x}\right| \leq \operatorname{Const}_{f} \cdot N^{9 / 4} \tau^{-N d(x)},
$$

where $\tau$ and the adaptive filter are given in Theorem 3.3 .

Although the optimal filter is equally applicable for pseudo-spectral data, as stated in the above theorem, computational efficiency can be gained by operating on the function's uniform samples in the physical space. In addition, operating directly on the samples circumvents going to the Fourier domain, and in doing so can avoid transferring information across the discontinuities.

\section{Optimal adaptive ORDER MOllifier}

As stated in equation (1.2), filtering can be applied as pointwise multiplication in the Fourier dual space, or by convolution with the mollifier in the physical space. In 93 we constructed and analyzed the optimal filter when the data is given as either the truncated Fourier coefficients or the function's uniform samples, Theorems 3.3 and 3.6. respectively. We now construct and analyze a mollifier defined in the physical space, using the optimal order filter's inverse Fourier transform, $\phi_{p, \gamma}(N \cdot)$, which similarly to the optimal filter satisfies optimal (in the sense of Property 2.2) joint time-frequency localization. We focus here on the mollifier applied to the pseudo-spectral data, where using a mollifier defined entirely in the physical space allows for a purely physical space implementation. We now detail the error analysis for the mollifier, $\phi_{p, \gamma}(N \cdot)$, proving the same convergence rate as in Theorem 3.6 .

Unlike the filter's associated mollifier, $\psi(\cdot)$, the mollifier defined directly in the physical space, $\phi_{p, \gamma}(N \cdot)$, is not periodic, and as such the implementation requires the periodic extension of the samples

$$
f_{\pi}\left(y_{\nu}+2 \pi n\right):=f\left(y_{\nu}\right) \quad \text { for } \quad n=-1,0,1 .
$$

Moreover we define the symmetric region containing the $2 N$ samples centered about $x$ as $I_{x}:=(x-\pi, x+\pi]$. 
The error after discrete mollification with $\phi_{p, \gamma}(N \cdot)$ is defined, and can be expanded 11 as follows:

$$
\begin{aligned}
E_{\mathrm{mol}}(N, x):= & f(x)-\frac{\pi}{N} \sum_{y_{\nu} \in I_{x}} f_{\pi}\left(y_{\nu}\right) \phi_{p, \gamma}\left(N\left(x-y_{\nu}\right)\right) \\
= & f(x)-\frac{\pi}{N} \sum_{y_{\nu} \in I_{x}} f_{\pi}\left(y_{\nu}\right) S_{N} \psi\left(x-y_{\nu}\right) \\
& +\frac{\pi}{N} \sum_{y_{\nu} \in I_{x}} f_{\pi}\left(y_{\nu}\right)\left[S_{N} \psi\left(x-y_{\nu}\right)-\psi\left(x-y_{\nu}\right)\right] \\
& +\frac{\pi}{N} \sum_{y_{\nu} \in I_{x}} f_{\pi}\left(y_{\nu}\right)\left[\psi\left(x-y_{\nu}\right)-\phi_{p, \gamma}\left(N\left(x-y_{\nu}\right)\right)\right] \\
= & : \mathcal{E}_{1}+\mathcal{E}_{2}+\mathcal{E}_{3} .
\end{aligned}
$$

The first component is simply the error when filtering the trigonometric interpolant,

$$
\begin{aligned}
\mathcal{E}_{1} & :=f(x)-\frac{\pi}{N} \sum_{y_{\nu} \in I_{x}} f_{\pi}\left(y_{\nu}\right) S_{N} \psi\left(x-y_{\nu}\right) \\
& =f(x)-\frac{1}{2 \pi} \sum_{|k| \leq N} \sigma\left(\frac{k}{N}\right) \tilde{f}_{k} e^{i k x} \\
& =f(x)-\psi * I_{N} f(x),
\end{aligned}
$$

which is composed of the localization and accuracy error components as in equation (3.17).

The second component of $E_{\mathrm{mol}}$ satisfies the same bound as the dual space localization error, $L_{D}(N, p, x)$,

$$
\begin{aligned}
\left|\mathcal{E}_{2}\right| & =\frac{\pi}{N}\left|\sum_{y_{\nu} \in I_{x}} f_{\pi}\left(y_{\nu}\right)\left[S_{N} \psi\left(x-y_{\nu}\right)-\psi\left(x-y_{\nu}\right)\right]\right| \\
& \leq 2 \pi\|f\|_{L^{\infty}[-\pi, \pi]}\left\|\psi-S_{N} \psi\right\|_{L^{\infty}} \\
& \leq \text { Const }_{f} \sum_{|k|>N} \sigma \frac{k}{N} \leq \text { Const }_{f} \int_{1}^{\infty} \sigma(\xi) d \xi \\
& \leq \text { Const }_{f}\left(\frac{p}{\gamma}\right)^{2}\left(\frac{\gamma^{2} e}{2 p}\right)^{p} e^{-\gamma^{2} / 2}
\end{aligned}
$$

and the third component is controlled by the different between the mollifiers, $\psi(\cdot)$ and $\phi_{p, \gamma}(N \cdot)$, which are exponentially close as quantified in equation (3.5)

$$
\begin{aligned}
\left|\mathcal{E}_{3}\right| & =\frac{\pi}{N}\left|\sum_{y_{\nu} \in I_{x}} f_{\pi}\left(y_{\nu}\right)\left[\psi\left(x-y_{\nu}\right)-\phi_{p, \gamma}\left(N\left(x-y_{\nu}\right)\right)\right]\right| \\
& \leq 2 \pi\|f\|_{L^{\infty}[-\pi, \pi]} \cdot\left\|\psi(z)-\phi_{p, \gamma}(N z)\right\|_{L^{\infty}[-\pi, \pi]} \\
& \leq \text { Const }_{f} \frac{\gamma p^{3 / 4}}{N^{2}} e^{-(\pi N / 2 \gamma)^{2}} .
\end{aligned}
$$

\footnotetext{
${ }^{11}$ The error expansion is achieved by adding and subtracting$$
\frac{\pi}{N} \sum_{y_{\nu} \in I_{x}} f_{\pi}\left(y_{\nu}\right)\left[S_{N} \psi\left(x-y_{\nu}\right)-\psi\left(x-y_{\nu}\right)\right]
$$

so as to decompose the error into terms bounded previously in 3 
Combining the above bounds we achieve the same overall bound as for the filtered spectral projection,

$$
\begin{aligned}
\left|E_{\mathrm{mol}}(N, x)\right| \leq & \left|f(x)-\psi * I_{N} f(x)\right| \\
& + \text { Const }_{f}\left[\left(\frac{p}{\gamma}\right)^{2}\left(\frac{\gamma^{2} e}{2 p}\right)^{p} e^{-\gamma^{2} / 2}+\frac{\gamma p^{3 / 4}}{N^{2}} e^{-(\pi N / 2 \gamma)^{2}}\right] \\
\leq & \text { Const }_{f}\left[r N \beta_{f}^{-p}+C_{1} e^{-\gamma^{2} / 2}+C_{2} e^{-(r N / 2 \gamma)^{2}}\right],
\end{aligned}
$$

where $C_{1}$ and $C_{2}$ are defined in equation (3.11). Being the same bound as was achieved for filtering the spectral projection, (3.10), the localization and accuracy parameters are selected in the same fashion as for the optimal adaptive order filter, $\gamma:=\sqrt{\alpha N d(x)}$ and $p:=\kappa N d(x)$. The above results are summarized in the following theorem:

Theorem 4.1. Given the $2 N$ equidistant samples of a piecewise analytic function, $\left\{f\left(\frac{\pi}{N}(\nu-N)\right)\right\}_{\nu=0}^{2 N-1}$, the function can be recovered within the exponential bound

$$
\left|f(x)-\frac{\pi}{N} \sum_{y_{\nu} \in I_{x}} f_{\pi}\left(y_{\nu}\right) \phi_{\mathrm{opt}}\left(N\left(x-y_{\nu}\right)\right)\right| \leq \operatorname{Const}_{f} \cdot N^{9 / 4} \tau^{-N d(x)},
$$

where $\tau:=\min \left(\beta_{f}^{k}, 2 e^{\alpha / 2}\left(\frac{\kappa}{\alpha e}\right)^{\kappa}, \frac{e^{1 / 2 \alpha}}{(1+\sqrt{2})^{\kappa}}\right)$, and the mollifier is given by

$$
\phi_{\text {opt }}(N, x):=\frac{1}{\sqrt{\alpha N d(x)}} \exp \left(\frac{-N x^{2}}{2 \alpha d(x)}\right) \sum_{n=0}^{\lfloor\kappa N d(x)\rfloor} \frac{4^{-n}}{n !} H_{2 n}\left(x \sqrt{\frac{N}{2 \alpha d(x)}}\right) .
$$

Again, convergence is ensured if the parameter constants satisfy

$$
\alpha \kappa<1 / 2 \ln (1+\sqrt{2}) \approx 0.56,
$$

and although their optimal values are function-dependent we propose $\alpha=1$ and $\kappa=1 / 15$ as reasonable function-independent values, in which case $\tau$ is given as before in equation (3.15). Also we reiterate Remark 3.5 that unlike the adaptive filter and mollifier of [24] and 23, modulo exponentially small differences, the optimal filter and mollifier are performing the same action in the dual and physical spaces. This is reflected in the mollifier dilation factor $\sqrt{d(x) / N}$ which is the inverse of the filter's dilation rate, as the Fourier transform would imply.

Remark 4.2. As has been shown, by abandoning compact support we are able to develop optimal filters and mollifiers which balance the order of the fundamental error contributions, and in doing so recover (pointwise) true exponential accuracy. However, in the discrete case maintaining compact support can be beneficial, because, unlike spectral data, the pseudo-spectral data (the function's uniform samples) have not intermixed information from different regions of smoothness. In addition, applying the mollifier directly to the uniform samples avoids computation of the pseudo-spectral coefficients. Consequently, if the mollifier is compactly supported to the region of smoothness, information will not be transfered across the discontinuity. Rather than construct a compactly supported mollifier directly (as was done in 23]), it is advantageous to use the optimal mollifier and truncate it to the region of smoothness. This is achieved by restricting the optimal mollifier to act on the samples in the sub-interval $I_{x, d(x)}:=(x-d(x), x+d(x))$. This truncation of the 
analytic optimal mollifier avoids transferring information across the discontinuity, yet the true exponential accuracy can be retained. The additional error incurred by truncating the mollifier is of the same size as the physical space localization error, and as such does not affect the order of the overall bound in Theorem 4.1 . Moreover, normalizing this truncated optimal mollifier to unit mass results in first order accuracy in the immediate neighborhood of the discontinuity, [23].

We note in passing that a similar analysis can be developed for the mollifier applied to a function's spectral projection directly in the physical space. However, such an analysis is omitted for conciseness and as this option is less efficient than applying the optimal filter in the Fourier space. Specifically, applying the mollifier in the physical space requires evaluation of the convolution which is computationally less efficient than pointwise multiplication of the filter directly in the Fourier space.

\section{Numerical EXAMPLES}

We begin with contrasting the optimal adaptive filter, $\sigma_{\text {opt }}(\cdot)$, and mollifier, $\phi_{\text {opt }}(\cdot)$, presented in Theorems 3.3 and 4.1 with the more traditional compactly supported adaptive filter and mollifier constructed and analyzed in [24] and [23],

$$
\begin{aligned}
& \sigma_{\text {adapt }}(\xi):=\left\{\begin{array}{ll}
e^{\left(\frac{c_{q} \xi^{q}}{\xi^{2}-1}\right)}, & |\xi|<1, \\
0, & |\xi| \geq 1,
\end{array} c_{q}:=2^{q} \frac{3}{8} \cdot \frac{18 q^{2}+3 q+14}{9 q^{2}+6 q+2},\right. \\
& \psi_{\text {adapt }}(x):= \begin{cases}\frac{2}{d(x)} \frac{\sin \left((p(x)+1) \frac{\pi x}{d(x)}\right)}{\sin \left(\frac{\pi x}{2 d(x)}\right)} \exp \left(\frac{10 x^{2}}{x^{2}-d(x)^{2}}\right), & |x|<d(x), \\
0, & |x| \geq d(x),\end{cases}
\end{aligned}
$$

with adaptive orders, $q(x):=\max \left(2, \frac{1}{2} \sqrt{N d(x)}\right)$, and $p(x):=N d(x) / \pi \sqrt{e}$. In 24 and 23 it was shown that the above compactly supported filter and mollifier satisfy theorems similar to Theorems 3.3 and 4.1 , but with the root-exponential convergence rate $\exp \left(-\eta_{f} \sqrt{N d(x)}\right)$ for some $\eta_{f}>0$, depending on the particular
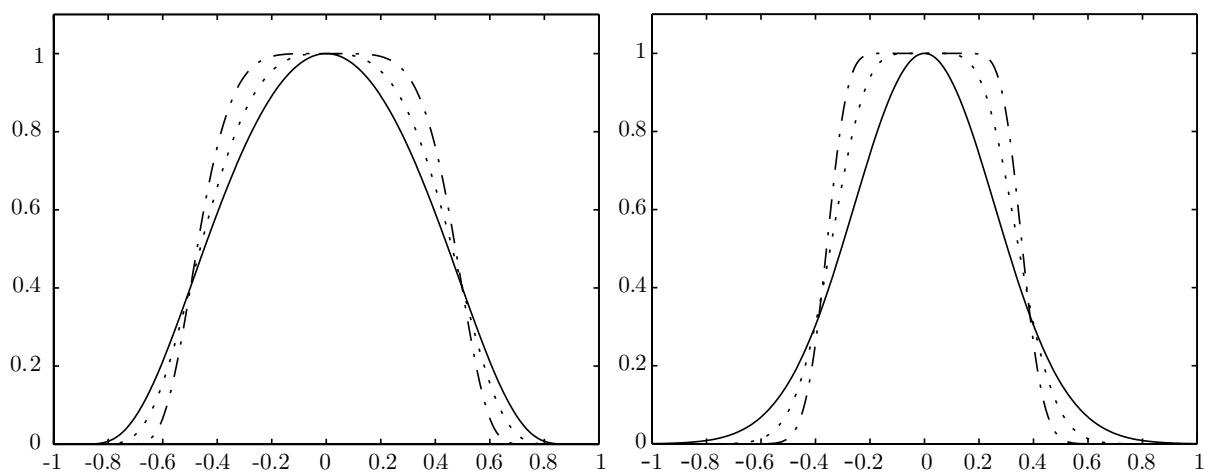

FiguRE 5.1. The compactly supported adaptive filter (5.1) shown left, and optimal filter (3.14) seen right, for $N=128$, and $d(x)=$ $3^{(n-3)} \pi$ with $n=0,1,2$, solid, dotted, and dashed, respectively. The resulting adaptive filter orders are, $q=2,3,5$ and the optimal filter of order $2 p$ with $p=0,2,8$. 


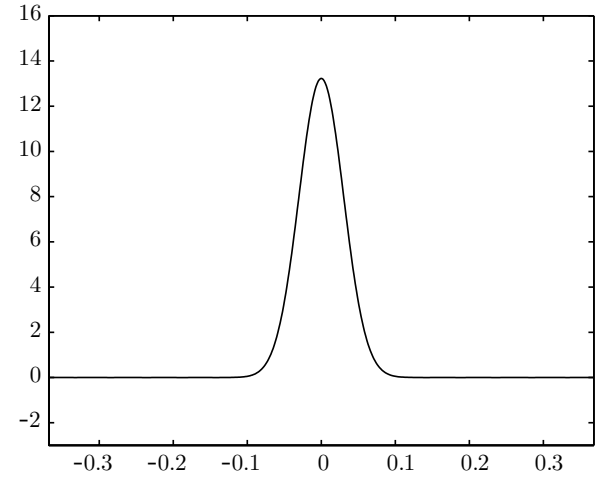

(a)

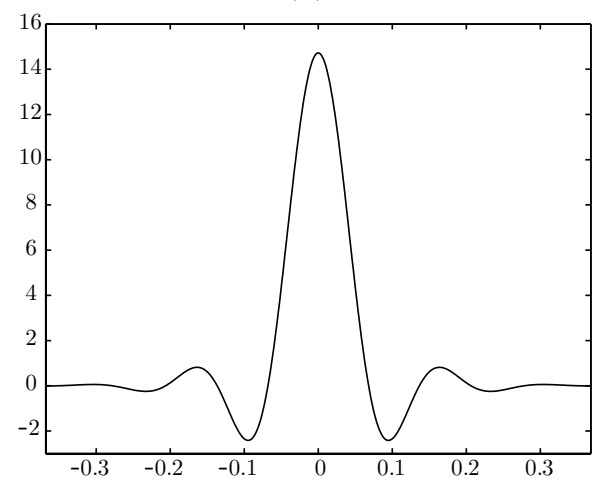

(c)

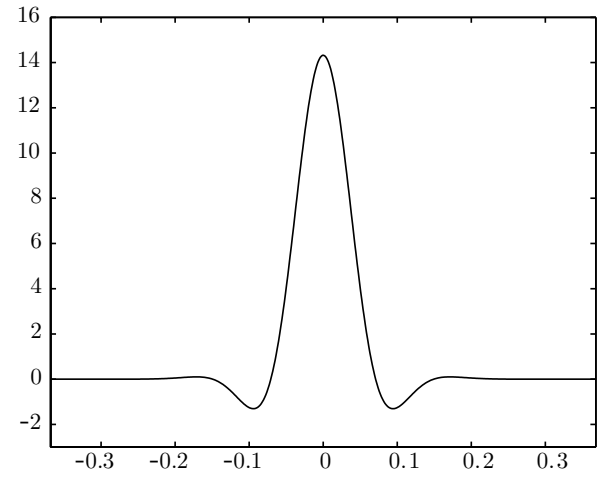

(b)

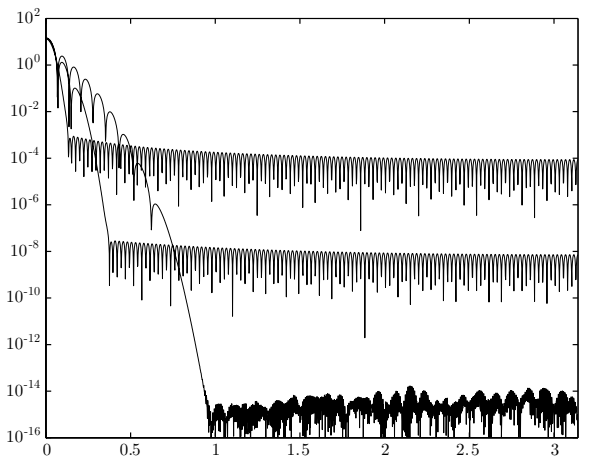

(d)

Figure 5.2. The optimal filter $\sigma_{\text {opt }}(\cdot)$ 's associated mollifier for $N=128$ and $d(x)=3^{(n-3)} \pi$ in (a-c) for $n=0,1,2$, respectively, and the $\log$ of the same mollifiers $(\mathrm{d})$ with increasing asymptotic decay for increasing $d(x)$.

function being filtered. The optimal filter and mollifier achieve superior, true exponential accuracy by not imposing compact support in the dual or physical space respectively; rather, they satisfy optimal joint time-frequency localization to

$$
[x-d(x), x+d(x)] \times[-N, N] .
$$

Figure 5.1 illustrates the adaptive filter of [24] and the optimal filter for a fixed value spectral projection order, $N$, and various values of $d(x)$. Throughout this section the optimal filter and mollifier use the functions-independent parameter values stated in equation (3.15).

Figure 5.2 shows the mollifiers associated with the optimal adaptive order filter, $\sigma_{\mathrm{opt}}$, shown in Figure 5.1(right). As the filter order increases, the number of near-vanishing moments increases, exhibited through increased oscillations and improved asymptotic physical space localization, but at the cost of decreased initial localization. By selecting the optimal filter parameters as (3.12), the optimal filter and mollifier balance these competing behaviors, resulting in the minimal error as decomposed in (2.3). 
The following numerical examples are performed for the function

$$
f(x)= \begin{cases}\left(2 e^{2 x}-1-e^{\pi}\right) /\left(e^{\pi}-1\right), & x \in[0, \pi / 2), \\ -\sin (2 x / 3-\pi / 3), & x \in[\pi / 2,2 \pi),\end{cases}
$$

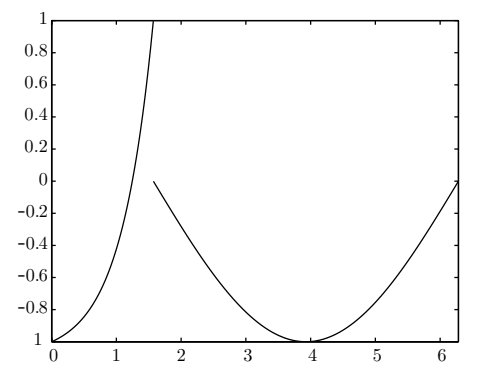

which was proposed in 23] as a challenging test problem with a large gradient to the left of the discontinuity at $x=\pi / 2$. Moreover, lacking periodicity $f(\cdot)$ feels three discontinuities per period,

$$
d(x)=\min (|x|,|x-\pi / 2|,|x-2 \pi|), \quad x \in[0,2 \pi],
$$

and has substantially different regularity constants for the two functions composing it which diminishes the potential for parameter tuning.

In Figure 5.3 the exact Fourier coefficients, $\left\{\hat{f}_{k}\right\}_{k \leq N}$, are given and then filtered to approximate $f(x)$. Although the adaptive filter achieves root-exponential convergence, Figure 5.3(c), it is at a substantially slower rate than is realized by the optimal order filter, Figure 5.3(d), which possesses true exponential convergence as stated in Theorem 3.3. Specifically, the asymptotic exponential convergence rate constant $\tau$ is seen to be approximately 1.37. Moreover, for the optimal filter the convergence in the immediate neighborhood of the discontinuities is also improved as illustrated in the removal of small oscillations seen in Figure 5.3(a) but not 5.3(b).

Figure 5.4 contrasts the adaptive mollifier of [23] with the optimal mollifier, (4.2), where the function $f(x)$ is approximated from its $2 N$ equidistant samples over $[0,2 \pi)$, through discrete physical space convolution as stated in Theorem 4.1 . The optimal adaptive order mollifier yields a nearly indistinguishable asymptotic convergence rate as the optimal filter, Figures 5.3(d) and 5.4(d). In addition, the optimal mollifier substantially out-performs the adaptive mollifier, (5.2), as contrasted in Figures 5.4(c), (d). For the above computations the optimal mollifier is applied only to the samples in the symmetric interval, $I_{x, d(x)}=(x-d(x), x+d(x))$, and both mollifiers are normalized to possess exact unit mass. By further limiting their support to contain at least two samples, $d(x):=\max (d(x), \pi / N)$, this normalization results in at least first-order approximations, significantly reducing the blurring in the immediate $\mathcal{O}(1 / N)$ neighborhood of the discontinuities, as contrasted in Figure 5.3(a), (b) and 5.4(a), (b). A full discussion of this normalization is given in [23. For the optimal filter to retain high order accuracy up to the discontinuity, a one-sided filter as described in 4. could be constructed from the optimal filter; for conciseness we do not pursue this avenue here.

Having established the significant improvements of the optimal filter and mollifier over the adaptive filter and mollifier, we now contrast these methods with the 


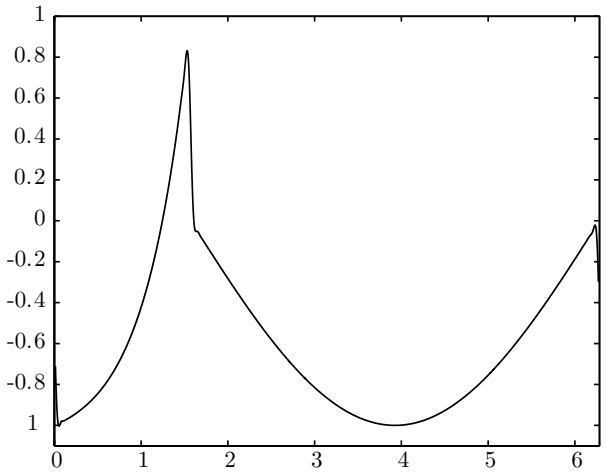

(a)

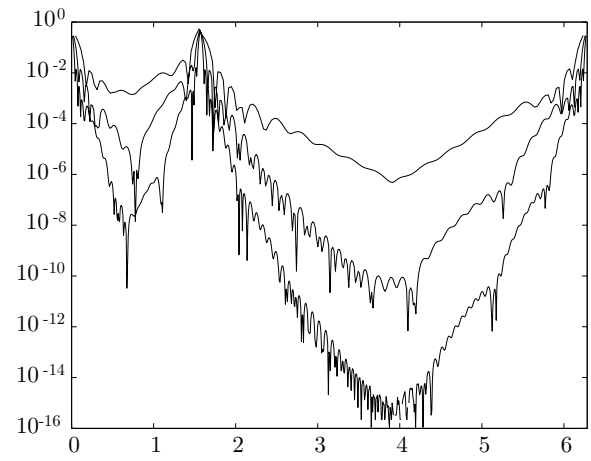

(c)

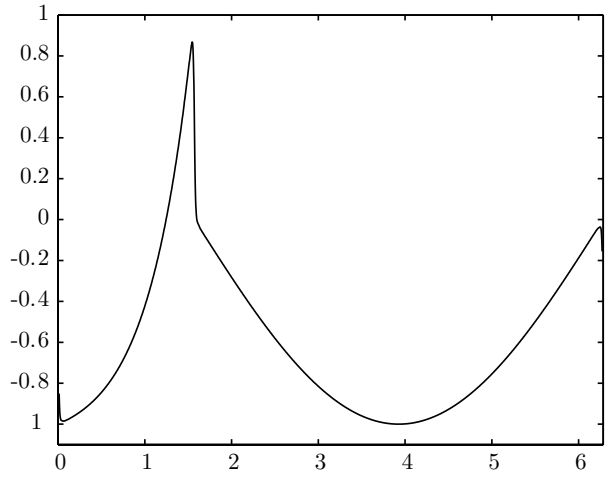

(b)

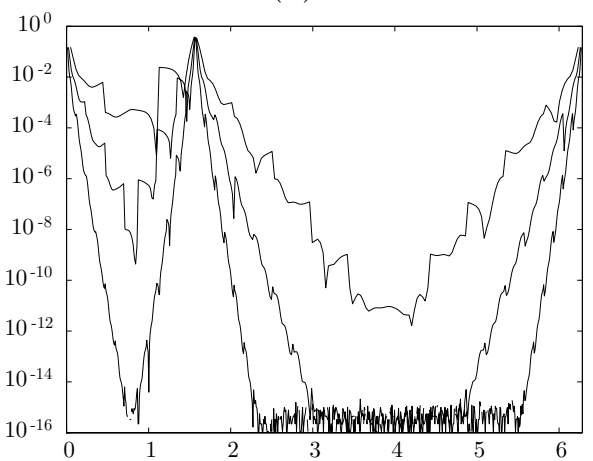

(d)

FIGURE 5.3. The filtered 128 mode spectral projection using the adaptive filter (5.1) and optimal filter (3.14), (a) and (b) respectively. The error in the reconstructions for $N=32,64,128$ for the adaptive filter (c) and optimal filter (d).

pioneering adaptive Erfc-Log filter of Boyd [2],

$$
\sigma_{e r f c}(\xi):=\frac{1}{2} \operatorname{erfc}\left\{2 \sqrt{m}(|\xi|-1 / 2) \sqrt{\frac{-\ln \left(1-4[|\xi|-1 / 2]^{2}\right)}{4[|\xi|-1 / 2]^{2}}}\right\},
$$

with the adaptive order $m(x):=1+N d(x) / 2 \pi$. The improved true exponential convergence rate of the optimal filter and mollifier is realized by abandoning the traditional construction with compact support, and developing analytic filters and mollifiers. Although the convergence properties of this Erfc-Log filter are much less understood, it is similarly analytic, and the numerical examples here exhibit its numerical effectiveness. The form of the Erfc-Log filter was developed as an asymptotic approximation of the Vandeven filter [25]. The adaptive order $m(x)$ was derived independently from Euler lag average acceleration (a piecewise constant approximation of filtering) applied to the sawtooth function, [1], and was put forth as the optimal adaptive order for general filters. Although correctly capturing the desired properties of an adaptive order, the transition from Euler lag averaging to general filters fails to capture the subtleties of the filter's smoothness. In particular, for infinitely differentiable compactly supported filters the optimal order is given as 


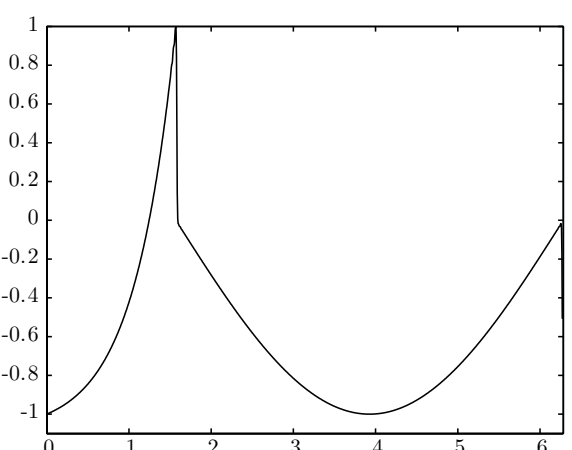

(a)

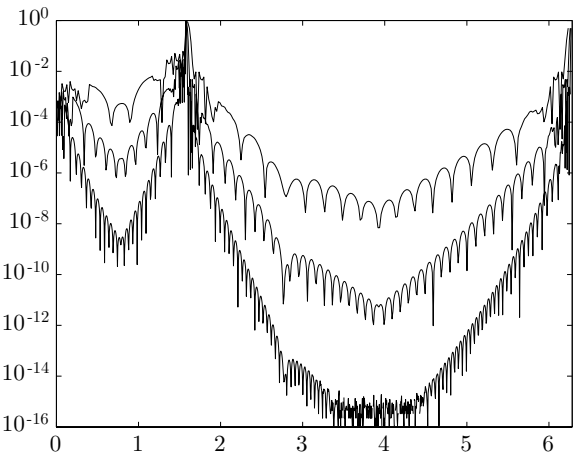

(c)

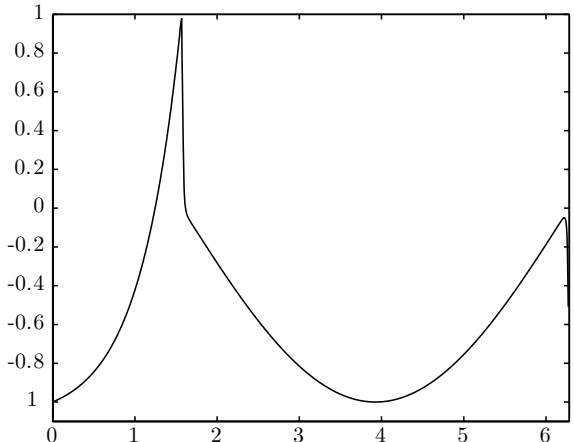

(b)

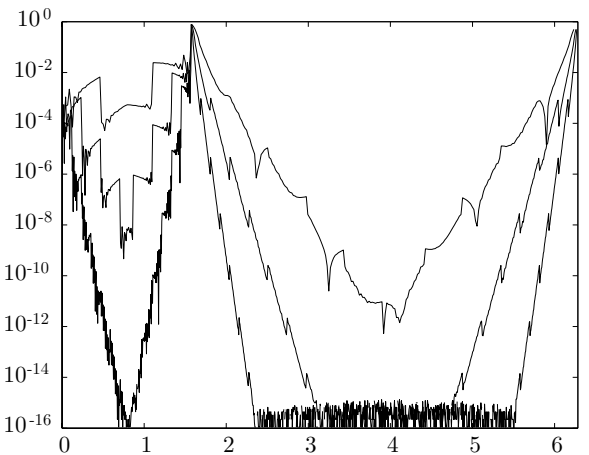

(d)

FiguRE 5.4. The reconstruction of (5.3) from its 256 uniform samples using the adaptive mollifier (5.2) and optimal mollifier (4.2) is shown in (a) and (b), respectively. Additionally, the error in the pseudo-spectral reconstructions with $N=32,64,128$ for the adaptive mollifier (c) and optimal mollifier (d) is illustrated.

a fractiona 12 power of $N d(x), 24$. Nevertheless, from the analysis developed in 24 and $\$ 3$ it can be conjectured that for analytic filters the order proportionality $N d(x)$ is indeed correct. The error decomposition developed here in (2.3) may allow for a rigorous analysis of the Erfc-Log filter and proof of exponential convergence.

Figure 5.5] directly contrasts the adaptive, Erfc-Log, and optimal filter (left) as well as the adaptive and optimal mollifier (right). True exponential accuracy is illustrated for the optimal and Erfc-Log filter, with the optimal filter achieving somewhat better results. The Erfc-Log filter consistently outperforms the adaptive filter, however it is worth noting that no proof of convergence is currently available for the Erfc-Log filter. A similar exponential convergence rate can be expected of the traditional exponential filter $\sigma_{\exp }(\xi):=\exp \left(-c \xi^{2 p}\right)$ with properly chosen

\footnotetext{
${ }^{12}$ Compactly supported infinitely differentiable functions can be analyzed in terms of their Gevrey regularity, which is categorized by the growth rate of a function's derivatives, $\left\|f^{(s)}\right\|_{L^{\infty}} \leq$ Const $\cdot(s !)^{\alpha} / \eta_{f}^{s}$ for $\alpha>1$. The resulting optimal adaptive filter order for Gevrey regular filters is $q(x) \sim(N d(x))^{1 / \alpha}$. For Gevrey regular functions replacing the root adaptive filter order with that arrived at through the Euler lag averaging, $\sim N d(x)$, results in a dramatically decreased convergence rate.
} 

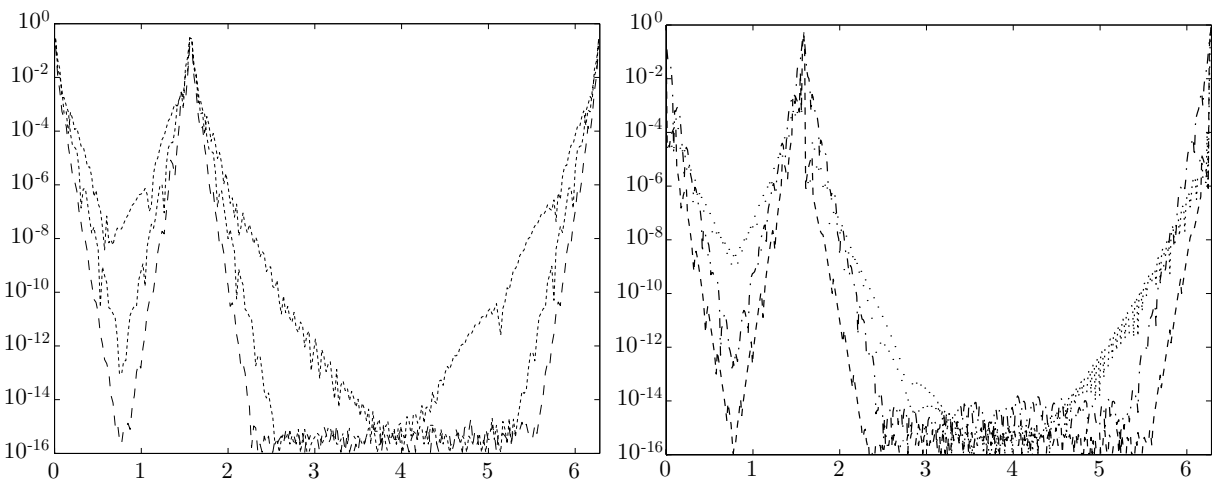

Figure 5.5. Left: The error for the filtered $N=128$ mode spectral projection of (5.3) using the adaptive (dots), Erfc-Log (dot dash), and optimal (dash) filters. Right: The error after approximating (5.3) from its 256 uniform samples using the adaptive (dots) and optimal (dash) mollifier. As there is no physical space, mollifier, analog to the Erfc-Log filter, for completeness the Erfc-Log filtered trigonometric interpolant (dot dash) is shown.

parameters, $c$, and $p \sim N d(x)$. However, as neither the complementary error function nor the exponential filter are known to in any way satisfy optimal joint time-frequency localization, there is little reason to believe they will be as accurate as the optimal filter.

The primary advantage of filtering over other methods for the resolution of Gibbs' phenomenon is their computational efficiency. Specifically, for nonadaptive filters $\mathcal{O}(N)$ points can be approximated within a $\mathcal{O}(N \log N)$ computation cost by utilizing the ubiquitous FFT algorithm. Although a faithful implementation of the optimal filter or mollifier requires $\mathcal{O}\left(N^{2}\right)$ computational cost to approximate $\mathcal{O}(N)$ points, the $\mathcal{O}(N \log N)$ efficiency can be recovered by using a tiered approach. Rather than utilizing a different filter for each point, the tiered approach designates a finite number of intervals in each of which a selection of the optimal filter is used. Unfortunately, as symmetric filters and mollifiers necessarily sacrifice accuracy when the discontinuities are approached, they cannot recover high order accuracy up to the discontinuities. Here we do not contrast the optimal filter and mollifier with the interval methods designed to recover high resolution up to the discontinuities (e.g., [3, 5, 6, 12, 14, 19]), but note that in [12] numerical experiments are performed on (5.3) allowing for a direct comparison. As stated earlier, the hybrid method [8] is a particularly computationally efficient synthesis of these interval methods and filtering. The hybrid method uses the tiered approach describe above, with one of the interval methods used for the intervals within a $\mathcal{O}(1 / N)$ neighborhood of the discontinuity.

\section{SUMMARY}

In this work we determine the fundamental error contributions for a general filter or mollifier. From this perspective we discard the classical restriction of compact support, and use the theory of time-frequency analysis to construct a filter and 
mollifier with optimal joint time-frequency localization to the region dictated by the function's smoothness, and the projection order, $[x-d(x), x+d(x)] \times[-N, N]$. This analysis results in the new fundamental dilation relationship $\sqrt{N / d(x)}$ and its inverse in the dual and physical spaces, respectively. Furthermore, similar to 23, 24 the number of near-vanishing moments is selected to balance the accuracy and localization errors, again determined to be a function of both localization parameters $N$ and $d(x)$. The significant improvements in the convergence rate, are reflected in Theorems 3.3 and 4.1 and are illustrated in Figures 5.3 and 5.4, In addition to the improved accuracy, the optimal filter and mollifier retain robustness to inaccuracies in the calculation of $d(x)$, as using an incorrect value does not destroy the accuracy for the entire smooth region. Rather, a sub-optimal exponential accuracy is realized due to the imbalance of the localization and accuracy errors. In conclusion, the optimal filter and mollifier presented here are a robust, exponentially accurate, and computationally efficient method for the manipulation of piecewise smooth functions, given its spectral information.

\section{ACKNOWLEDGMENTS}

I would like to thank Thomas Strohmer for the introduction to Hermite Distributed Approximating Functionals, as well as to thank Anne Gelb, Roland Wilson, David Gottlieb, and John Boyd for numerous useful comments on the exposition.

\section{REFERENCES}

1. J. P. Boyd, A Lag-Averaged Generalization of Euler's Method for Accelerating Series, Appl. Math. Comput., (1995) 143-166. MR1346575 (96d:65007)

2. J. P. Boyd, The Erfc-Log Filter and the Asymptotics of the Euler and Vandeven Sequence Accelerations, Proceedings of the Third International Conference on Spectral and High Order Methods, (1996) 267-276.

3. O.P. Bruno, Fast, High-Order, High-Frequency Integral Methods for Computational Acoustics and Electromagnetics, Topics in Computational Wave Propagation Direct and Inverse Problems Series: Lecture Notes in Computational Science and Engineering, 31 (M. Ainsworth, P. Davies, D. Duncan, P. Martin, B. Rynne, eds.), Springer, 2003, 43-83. MR.2032867 (2004k:65260)

4. W. Cai, D. Gottlieb, and C.W. Shu, On One-Sided Filters for Spectral Fourier Approximations of Discontinuous Functions, SIAM Journal of Numerical Analysis, 29 (1992) 905-916. MR.1173176 (93e:65021)

5. T.A. Driscoll and B. Fornberg, A Padé-Based Algorithm for Overcoming the Gibbs' Phenomenon, Num. Alg. 26 (2001) 77-92. MR1827318 (2002b:65007)

6. K.S. Eckhoff, On a High Order Numerical Method for Functions with Singularities, Math. Comp. 67(223) (1998) 1063-1087. MR.1459387 (98j:65014)

7. A. Gelb, The resolution of the Gibbs phenomenon for spherical harmonics, Math. Comp. 66 (1997) 699-717. MR1401940 (97k:42005)

8. A. Gelb, A Hybrid Approach to Spectral Reconstruction of Piecewise Smooth Functions, Journal of Scientific Computing, October 2000. MR.1828746 (2002a:65028)

9. A. Gelb and Zackiewicz, Determining Analyticity for Parameter Optimization of the Gegenbauer Reconstruction Method, preprint.

10. A. Gelb and E. Tadmor, Detection of Edges in Spectral Data, Applied Computational Harmonic Analysis 7 (1999) 101-135. MR.1699594 (2000g:42003)

11. A. Gelb and E. Tadmor, Detection of Edges in Spectral Data II. Nonlinear Enhancement, SIAM Journal of Numerical Analysis 38 (2000) 1389-1408. MR.1790039 (2001i:42003)

12. A. Gelb and J. Tanner, Robust Reprojection Methods for the Resolution of the Gibbs Phenomenon, ACHA 20 (1), (2006) 3-25. 
13. D. Gottlieb and C.-W. Shu, On The Gibbs Phenomenon IV: recovering exponential accuracy in a sub-interval from a Gegenbauer partial sum of a piecewise analytic function, Math. Comp. 64 (1995) 1081-1095. MR.1284667 (97b:42004)

14. D. Gottlieb and C.-W. Shu, On the Gibbs phenomenon and its resolution, SIAM Review 39 (1998) 644-668. MR 1491051 (98m:42002)

15. D. Gottlieb and E. Tadmor, Recovering pointwise values of discontinuous data within spectral accuracy, in "Progress and Supercomputing in Computational Fluid Dynamics", Proceedings of 1984 U.S.-Israel Workshop, Progress in Scientific Computing, Vol. 6 (E. M. Murman and S. S. Abarbanel, eds.), Birkhauser, Boston, 1985, 357-375. MR0935160 (90a:65041)

16. I. Gradshteyn and I. Ryzhik, Table of Integrals, Series, and Products, Academic Press, 2000. MR.1773820 (2001c:00002)

17. K. Gröchenig, Foundations of Time-Frequency Analysis, Birkhäuser, Boston, 2001. MR.1843717 (2002h:42001)

18. D.K. Hoffman and D.J. Kouri, Hierarchy of Local Minimum Solutions of Heisenberg's Uncertainty Principle, Phy. Rev. Lett. 25 (2002) 5263-5267. MR.1813690 (2001k:81006)

19. J.-H. Jung and B.D. Shizgal, Generalization of the inverse polynomial reconstruction method in the resolution of the Gibbs' phenomenon, J. Comput. Appl. Math. 172 (2004) 131-151. MR2091135 (2005d:42002)

20. J.C. Mason and D.C. Handscomb, Chebyshev Polynomials, Chapman \& Hall/CRC, New York, 2003. MR1937591 (2004h:33001)

21. A. Majda, J. McDonough and S. Osher, The Fourier method for nonsmooth initial data, Math. Comput. 30 (1978) 1041-1081. MR0501995 (80a:65197)

22. E. Tadmor, Spectral Methods for Hyperbolic Problems, from "Lecture Notes Delivered at Ecole Des Ondes", January 24-28, 1994. Available at http://www.math.ucla.edu/ tadmor/pub/ spectral-approximations/Tadmor.INRIA-94.pdf

23. E. Tadmor and J. Tanner, Adaptive Mollifiers - High Resolution Recovery of Piecewise Smooth Data from its Spectral Information, J. Foundations of Comp. Math. 2 (2002) 155189. MR 1894374(2003b:42009)

24. E. Tadmor and J. Tanner, Adaptive Filters for Piecewise Smooth Spectral Data, IMA J. Numerical Analysis, 25 No. 4 (2005) 535-647.

25. H. Vandeven, Family of Spectral Filters for Discontinuous Problems, Journal of Scientific Computings, 6 No. 2 (1991) 159-192. MR1140344 (92k:65006)

Department of Statistics, Stanford University, Stanford, California 94305-9025

E-mail address: jtanner@stat.stanford.edu 